\title{
VACANCY CLUSTER DISTRIBUTIONS IN He IMPLANTED SILICON STUDIED BY SLOW POSITRON ANNIHILATION SPECTROSCOPY
}

\author{
R.S. BRUSA \\ Istituto Nazionale per la Fisica della Materia, Dipartimento di Fisica \\ Universita' di Trento 38050 Povo TN, Italy
}

\begin{abstract}
Doppler broadening measurements performed by a slow positron. beam on $p$-type Si samples implanted with $\mathrm{He}$ at $20 \mathrm{keV}$ and at a fluence of $5 \times 10^{15}$ and $2 \times 10^{16} \mathrm{~cm}^{-2}$ are reviewed and distussed. The evolution of the open volume defects distribution was studied as a function of isochronal and isothermal annealing of the samples. In the as implanted samples the majority of the open volume defects produced by implantation was passivated by $\mathrm{He}$. The open volume defects density decreases, reaching a minimum at $250^{\circ} \mathrm{C}$. In the $250-650^{\circ} \mathrm{C}$ temperature range there is an increase in defects due to the appearance of vacancy clusters. At the higher annealing temperatures $\left(700-900^{\circ} \mathrm{C}\right)$ the vacancy clusters disappear only in the samples implanted at $5 \times 10^{15} \mathrm{~cm}^{-2}$.
\end{abstract}

PACS numbers: $71.60 .+\mathrm{z}, 78.70 . \mathrm{Bj}, 71.55 . \mathrm{Cn}$

\section{Introduction}

In the last two years there was a novel interest in the production of clean cavities in silicon samples at well-defined depths. These cavities, with a mean diameter in the nanometer range, were found to be very useful in gettering metal contaminants that reduce the performances and the reproducibility of semiconductors devices. The recipe to obtain nanocavities in Si has been known since 1987 [1]: $\mathrm{He}$ implanted in Si at a high dose, coalesces forming bubbles and then, after annealing at temperature higher than $800^{\circ} \mathrm{C}$, out-diffuses leaving empty voids. The formation of helium bubbles and their evolution in cavities was studied [2] as a function of the helium dose and of the implantation energy. The density and the diameter of the voids were investigated by transmission electron microscopy (TEM) after different thermal processes. Very little was known about the atomic processes involved in the clustering of $\mathrm{He}$, and the role of $\mathrm{He}$ interaction with vacancy and vacancy clusters in cavity formation. Recently, the subject was systematically investigated with several techniques applied at samples implanted at a He fluence below $\left(5 \times 10^{15} \mathrm{~cm}^{-2}\right)$ and above $\left(2 \times 10^{16} \mathrm{~cm}^{-2}\right)$ the threshold for cavities formation. Information about the evolution of $\mathrm{He}$ and of the displaced 
silicon atoms distributions was obtained by elastic recoil detection (ERD) and Rutherford backscattering in channelling (RBS-C) [3, 4]. Thermal programmed desorption (TPD) [5] was employed to obtain information about thermodynamics and kinetics quantity. TEM was used on selected samples to identify the nanostructures. The distribution of open volume defects was found by positron annihilation spectroscopy (PAS) $[6,7]$.

\section{Experimental}

The samples, obtained from high purity $p$-type $(1.7-2.5 \Omega \mathrm{cm})$ silicon wafers, Czochralski grown, (100) oriented, were kept at liquid nitrogen temperature and implanted at $20 \mathrm{keV}$ with an He fluence of $5 \times 10^{15}$ and $2 \times 10^{16} \mathrm{~cm}^{-2}$. Four series of samples were prepared and measured. Two series, one implanted at $5 \times 10^{15} \mathrm{He} / \mathrm{cm}^{2}$, the other implanted at $2 \times 10^{16} \mathrm{He} / \mathrm{cm}^{2}$, were isochronal annealed for two hours in the $150-900^{\circ} \mathrm{C}$ temperature range. Two series were treated isothermally at $250^{\circ} \mathrm{C}$ in the $2 \mathrm{~min}-24 \mathrm{~h}$ time range. By Monte-Carlo simulation the He projected range $R_{\mathrm{p}}$ was found to be about $230 \mathrm{~nm}$ with a straggling $\Delta R_{\mathrm{p}}$ of $85 \mathrm{~nm}$ [3].

The Doppler broadening measurements were performed with an electrostatic slow positron beam [8] coupled with a high purity germanium detector. The measured momentum distribution of the electron-positron annihilating pairs was characterized by the $S$ parameter calculated as the ratio of the counts in the central area of the peak and the total area of the peak. The narrowing of the momentum distribution (an increase in the $S$ parameter) is an indication of positron annihilation in open volume defects. In the analysis of PAS data we have also utilized the wing parameter $W$ that is the relative fraction of the counts in the wings region of the annihilation line. The $S$ and $W$ parameters were measured as a function of the positron implantation energy in the $0.06-25 \mathrm{keV}$ energy range. $S$ vs. $E$ data were analyzed by the diffusion model [9] and the $W(E)$ vs. $S(E)$ data by the method described in Ref. [10] to extract information about the distribution and the types of the open defects.

\section{Results and discussion}

As an example some selected $S$ parameter measurements, normalized to the silicon bulk $S$ value, are shown in Fig. 1 as a function of positron implantation energy and of the mean positron implantation depth. The lines are the best fit obtained by the diffusion equation model. The presence of open volume defects in the as implanted sample is evident from the figure. The number of defects increases in the sample annealed at $500^{\circ} \mathrm{C}$ and decreases, moving more deeper, in the sample annealed at $850^{\circ} \mathrm{C}$.

The density $N_{n v}$ of open volume defects and the mean depth of the defects distribution $\bar{d}$, as obtained by the best fitting procedure, are summarized in Fig. 2a and b, respectively, for the two series of samples isochronically annealed. Every type of open volume defect is characterized by a typical $S_{d}$ parameter. The $S_{d}$ values were obtained $[6,7]$ by the best fitting procedure and by the method of Ref. [10]. It was found that the characteristic $S_{d}$ values change with the thermal 


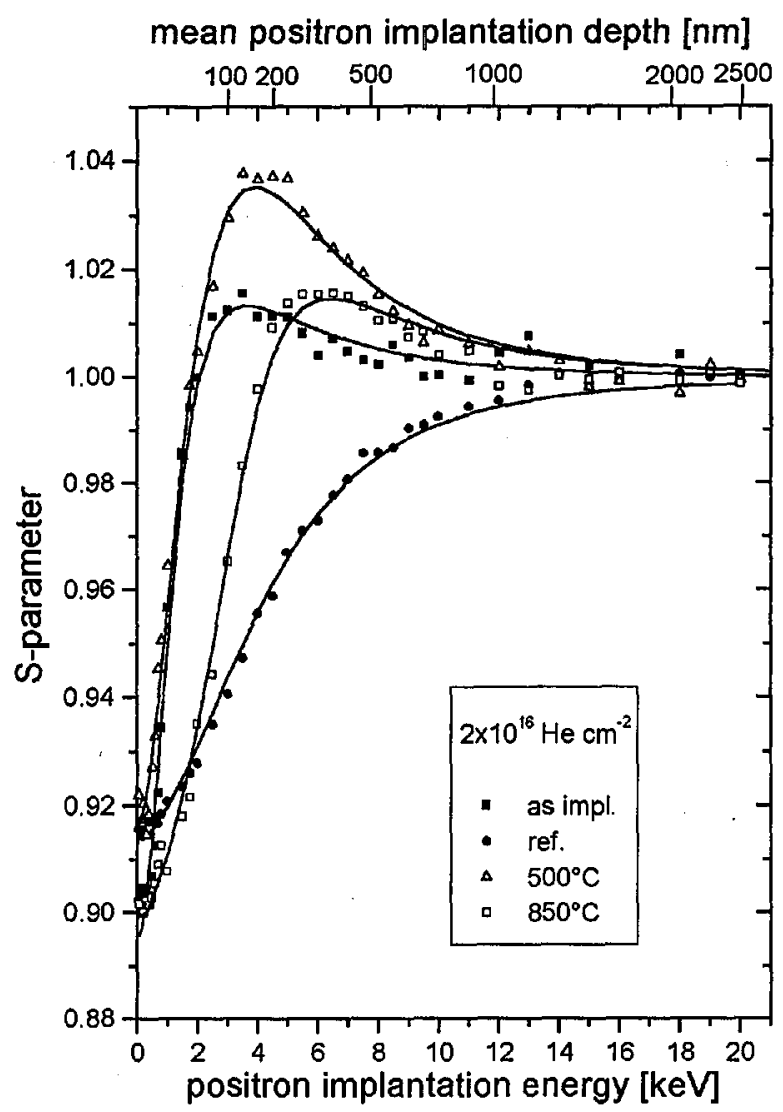

Fig. 1. $S$ parameter vs. positron implantation energy (lower scale) and the mean positron implantation depth (upper scale). Reference sample: full circles; sample implanted at a $2 \times 10^{16} \mathrm{~cm}^{-2}$ fluence of helium at $20 \mathrm{keV}$ : full squares; sample annealed at $500^{\circ} \mathrm{C}$ : open triangles; sample annealed at $850^{\circ} \mathrm{C}$ : open squares. The lines through the experimental points are best fit curves obtained according to the diffusion model.

treatment of the samples implanted at the two He doses. The increase in $S_{d}$ with the annealing temperature was an indication of positron trapping in larger open volume defects. $S_{d}$ reached the 1.112 value in correspondence with the positron annihilation in these samples (implanted at $2 \times 10^{16} \mathrm{He} / \mathrm{cm}^{2}$ and annealed at temperatures higher than $700^{\circ} \mathrm{C}$ ) in which nanocavities were observed by TEM measurements. The defects were classified by comparing the $S_{d}$ values with the $S_{d}$ values evaluated theoretically [11] for electron-positron pairs annihilating at ideal vacancy clusters in $\mathrm{Si}\left(V_{1}, \ldots, V_{5}\right.$ : monovacancy, .., pentavacancy). The helium related defects as measured by PAS were classified as $V_{1}^{*}, \ldots, V_{5}^{*}$ with the meaning of a distribution of different defects with a predominance of open volume defects with $V_{1}, \ldots, V_{5}$ character [7].

The discussion will be made with reference to Fig. 2. After implantation the majority of open volume defects are passivated by He atoms and involved in the 

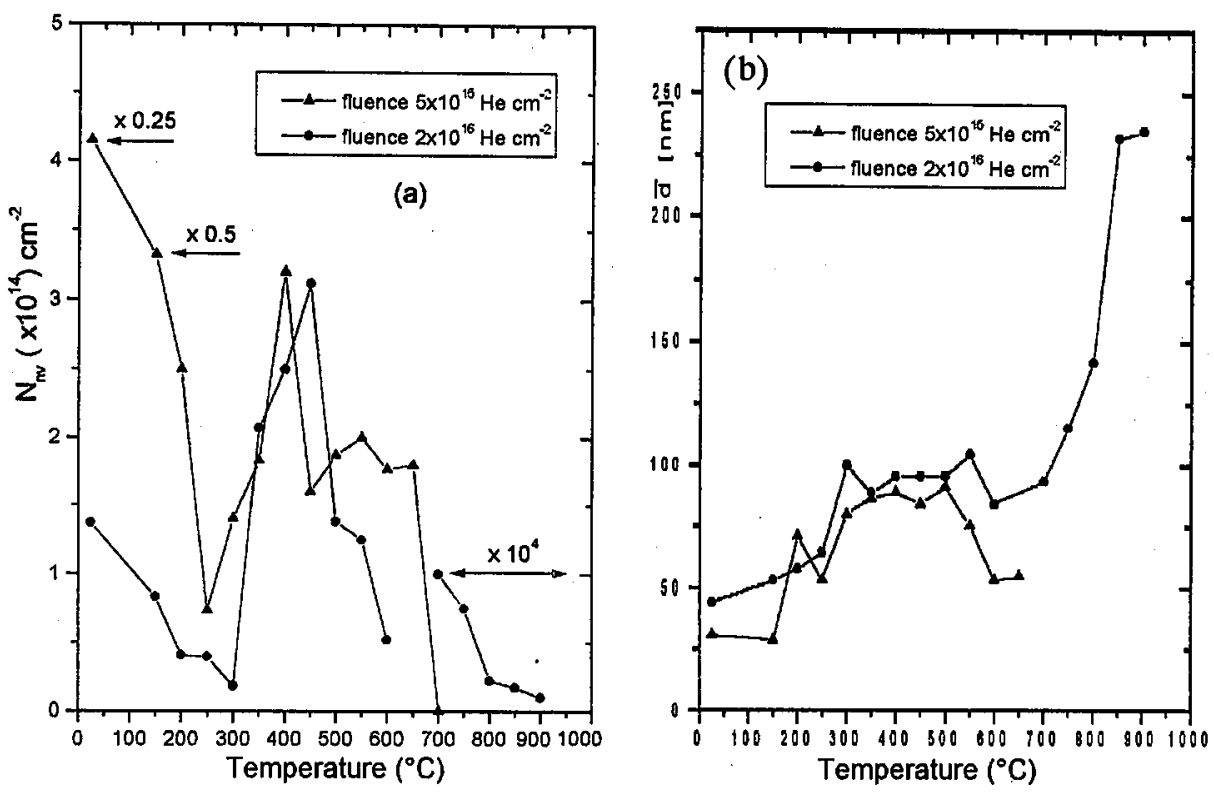

Fig. 2. Positron traps density $N_{n v}$ (a) and positron traps distribution mean depth $\bar{d}$ (b) as evaluated by fitting the $S$ vs. $E$ data with the diffusion model. The errors on $N_{n v}$ are $\pm 32 \%$ and on $\bar{d}- \pm 10 \%$.

He clustering [12]. In the samples implanted at low fluence $\left(5 \times 10^{15} \mathrm{He} \mathrm{cm}^{-2}\right)$ more $V_{1}^{*}\left(S_{d}=1.02\right)$ survived; contrary to it, in the sample implanted at high fluence $\left(2 \times 10^{16} \mathrm{He} / \mathrm{cm}^{2}\right)$ more $V_{2}^{*}\left(S_{d}=1.038\right)$ has been found. The mean depth distributions of $V_{1}^{*}$ and $V_{2}^{*}$ are at a depth more than five times less than the mean depth of the He distribution $(230 \mathrm{~nm})$ and three times less than the mean depth of the displaced silicon atoms distribution $(130 \mathrm{~nm})$. PAS reveals fewer defects in the sample implanted at the highest fluence: this can be due to a more effective passivation of the positron traps by He.

In the first stage of the thermal treatment $\left(150-250^{\circ} \mathrm{C}\right)$, according to ERD and TPD measurements, there is a negligible loss of helium from the samples. The content of $V_{1}^{*}$ and $V_{2}^{*}$ decreases. This decrease is due to passivation by $\mathrm{He}$ that moves towards the surface. $V_{1}^{*}$ completely disappears at $250^{\circ} \mathrm{C}$ and only $V_{2}^{*}$ remains. A part of the more unstable $V_{1}^{*}$ could also coalesce, during annealing, in $V_{2}^{*}$. The behavior of the open volume defects is similar in the samples annealed isothermally at $250^{\circ} \mathrm{C}$. In the first five minutes, when the helium loss is not observed, there is a decrease in $V_{1}^{*}$ and $V_{2}^{*}$, then $V_{2}^{*}$ starts to increase.

From $300^{\circ} \mathrm{C}$ the number of defect increases in both the series. In the $5 \times 10^{15} \mathrm{~cm}^{-2}$ series $V_{2}^{*}$ increases up to $400^{\circ} \mathrm{C}$, then $V_{4}^{*}\left(S_{d}=1.061\right)$ appears at $450^{\circ} \mathrm{C}$. The $V_{4}^{*}$ concentration decreases from $450^{\circ} \mathrm{C}$. At $700^{\circ} \mathrm{C}$ all defects are recovered. The mean depth of the defect distribution is about $85 \mathrm{~nm}$ from 300 to $500^{\circ} \mathrm{C}$, always less deeper than the mean depth of He distribution. In the $2 \times 10^{16}$ series the $V_{2}^{*}$ concentration increases from 300 to $350^{\circ} \mathrm{C}$ then remains about con- 
stant up to $450^{\circ} \mathrm{C}$. From $450^{\circ} \mathrm{C}$ there is the appearance of larger open volume defects $\left(V_{3}^{*}, S_{d}=1.052 ; V_{4}^{*}, S_{d}=1.061 ; V_{6}^{*}, S_{d}=1.088\right)$. In this temperature range the defects distribution moves towards a mean depth of about $95 \mathrm{~nm}$. The formation of these vacancy clusters is strictly related to the out-diffusion of $\mathrm{He}$ trapped in stronger defects [5]. At the annealing temperature of $700^{\circ} \mathrm{C}$ there is the appearance of the first cavities $\left(S_{d}=1.0112\right)$ confirmed by TEM measurements. From this annealing temperature the smaller clusters $\left(V_{4}^{*}, V_{6}^{*}\right)$ disappear and the $\mathrm{He}$ depletion is complete around $800^{\circ} \mathrm{C}$ [3]. The formation of cavities is activated by the He detrapping from big agglomerates but the increase in the size of cavities is also due to migration and coalescence of open volume defects [2]. TEM analysis has shown an increase in the mean radius of the cavities and the analysis of PAT data - a decrease in the cavity densities. The mean depth of the positron trap distribution moves from 100 to $230 \mathrm{~nm}$.

\section{Acknowledgments}

The author would like to thank A. Zecca and G.P. Karwasz for discussions, $\mathrm{N}$. Tiengo for the assistance in the experimental positron measurements, M. Bettonte for technical support, and G. Ottaviani and F. Corni for useful discussions about RBS, ERD, TPD results.

\section{References}

[1] C.C. Griffioen, J.H. Evans, P.C. De Jong, A. van Veen, Nucl. Instrum. Methods Phys. Res. Sec. B 27, 417 (1987).

[2] V. Raineri, P.G. Fallica, G. Pecorella, A. Battaglia, M. Barbagallo, S.U. Campisano, J. Appl. Phys. 78, 3727 (1995).

[3] R. Tonini, F. Corni, S. Fabbroni, G. Otta viani, G. Cerofolini, J. Appl. Phys. 84, 4802 (1998).

[4] F. Corni, G. Calzolari, S. Fabbroni, C. Nobili, G. Ottaviani, R. Tonini, G.F. Cerofolini, D. Leone, M. Servidori, R.S. Brusa, G.P. Karwasz, N. Tiengo, A. Zecca, J. Appl. Phys. 85, 1401 (1999).

[5] F. Corni, C. Nobili, G. Otta viani, R. Tonini, G. Calzolari, G.F. Cerofolini, G. Queirolo, Phys. Rev. B 56, 7331 (1997).

[6] R.S. Brusa, G.P. Karwasz, N. Tiengo, A. Zecca, F. Corni, G. Calzolari, C. Nobili, J. Appl. Phys. 85, 2309 (1999).

[7] R.S. Brusa, G.P. Karwasz, N. Tiengo, A. Zecca, F. Corni, R. Tonini, G. Ottaviani, submitted to Phys. Rev. B.

[8] A. Zecca, M. Bettonte, J. Paridaens, G.P. Karwasz, R.S. Brusa, Meas. Sei. Technol. 9, 1 (1998).

[9] A. Dupasquier, G. Ottaviani, in: Positron Spectroscopy of Solids, Eds. A. Dupasquier, A.P. Mills Jr., North-Holland, Amsterdam 1995, p. 581.

[10] M. Clement, J.M.M. de Nijs, P. Balk, H. Shut, A. van Veen, J. Appl. Phys. 79, 9029 (1996).

[11] M. Hakala, M.J. Puska, R.M. Nieminen, Phys. Rev. B 57, 7621 (1998).

[12] M. Alatalo, M.J. Puska, R.M. Nieminen, Phys. Rev. B 46, 12806 (1992). 